Revue internationale P.M.E.

\title{
Entre théorie et pratique
}

\section{Pierre-André Julien}

Volume 28, numéro 3-4, 2015

URI : https://id.erudit.org/iderudit/1035406ar

DOI : https://doi.org/10.7202/1035406ar

Aller au sommaire du numéro

Éditeur(s)

Editions EMS - In Quarto SARL

ISSN

0776-5436 (imprimé)

1918-9699 (numérique)

Découvrir la revue

Citer ce document

Julien, P.-A. (2015). Entre théorie et pratique. Revue internationale P.M.E., 28(3-4), 7-13. https://doi.org/10.7202/1035406ar d'utilisation que vous pouvez consulter en ligne.

https://apropos.erudit.org/fr/usagers/politique-dutilisation/ 


\title{
CHRONIQUE SUR LE MÉTIER DE CHERCHEUR
}

\section{Pierre-André JULIEN}

INRPME, Université du Québec à Trois-Rivières

\section{ENTRE THÉORIE ET PRATIQUE}

\begin{abstract}
Ceux qui cultivent les sciences chez les peuples démocratiques craignent toujours de se perdre dans les utopies. Ils se défient des systèmes, ils aiment à se tenir très près des faits et à les étudier par eux-mêmes; comme ils s'en laissent moins imposer facilement par le nom d'aucun de leurs semblables, ils ne sont jamais disposés à jurer de la parole du maître... ils pénètrent, autant qu’ils le peuvent, jusqu'aux parties principales du sujet qui les occupent... Les sciences ont alors une allure plus libre et plus sûre, et moins haute.
\end{abstract}

Alexis de Tocqueville De la démocratie en Amérique, 1835.

Toute théorie sur laquelle s'appuie une science doit absolument être mise à l'épreuve de la réalité de façon à pouvoir l'enrichir ou la rejeter, comme l'explique Karl Popper (1965). Ce que les philosophes, comme Bergson (1907/1969), appellent les recoupements et le va-et-vient nécessaire entre la déduction et l'induction, soit la meilleure façon de faire de la recherche, ou ce que Peirce (1896/1994) appelle l'abduction. C'est, évidemment, aussi le cas en PME et en entrepreneuriat.

\section{LES RAISONS POUR LIER LA THÉORIE Ȧ LA PRATIQUE EN PME ET EN ENTREPRENEURIAT}

Il existe au moins trois bonnes raisons pour les chercheurs dans cette science ${ }^{1}$ en développement de lier la pratique à la théorie et donc de faire systématiquement des vérifications sur le terrain. La première est que la ou les théories qui la soutiennent sont encore trop récentes, et même trop fragiles sur plusieurs aspects, pour penser qu'elle puisse se libérer des études sur le terrain, à l'encontre, par exemple, des analyses boursières comme celles des prix Nobel d'Économie $^{2}$, tel Lucas expliquant qu'on pouvait anticiper rationnellement les fluctuations

1 Si c'est vraiment une science, puisque beaucoup en doute !

2 Ou plutôt le prix de la Banque centrale de Suède en sciences économiques administré par la Fondation Nobel depuis sa création en 1969 après un fort lobbyisme, notamment de Gunnar Myrdal, à l'époque seul membre économiste de cette fondation, et qui a obtenu ce prix par la suite avec l'Allemand Friedrich von Hayek en 1974. 
boursières avec certains algorithmes, en se trompant pourtant lourdement, ou encore des applications de la théorie des jeux, comme c'est le cas du Français Jean Tirole ${ }^{3}$, qui ont manifestement peu à voir avec la réalité complexe. C'est d'ailleurs ce qu'ont fait les précurseurs comme de Serres, Cantillon, Say, Turgot ou Schumpeter, les trois premiers ayant écrit leur ouvrage à partir de leurs propres expériences en affaires, alors que les deux autres ont été ministres de l'Économie dans des périodes où les PME étaient vues comme le moteur de l'économie, du moins dans sa première période dans le cas de Schumpeter, à l'instar du temps d'Adam Smith.

La deuxième raison est que cette réalité est très complexe et changeante, et que, pour bien comprendre cette complexité issue notamment de la très grande hétérogénéité des PME et de leur évolution souvent fort diverse, il faut tenir compte des différentes tailles (travailleurs autonomes, micros, petites, moyennes entreprises), des secteurs et surtout des sous-secteurs, avec des productions souvent très pointues (par exemple, portant pour tout le produit ou pour quelques parties seulement) et divers types de marchés (très proches ou locaux, régionaux, nationaux, internationaux), ou encore de leurs besoins spécifiques en connaissance et de leur environnement aux ressources disponibles fort disparates. À l'encontre des grandes entreprises et de leurs énormes moyens et dont les dirigeants proviennent des mêmes grandes écoles avec un enseignement qui s'appuie trop souvent sur les mêmes modes, comme l'a noté Henry Mintzberg (1994) ${ }^{4}$, et qui s'échangent leurs dirigeants pour mieux assurer leur coopération dans la défense de leurs intérêts vis-à-vis des gouvernements et des populations ${ }^{5}$. On sait que les très petites entreprises, notamment les travailleurs autonomes, comptant ensemble pour plus des trois quarts des entreprises, agissent de façon fort différente selon la psychologie, l'expérience ou le métier et, bien sûr, les intérêts de leurs dirigeants. De plus, elles se distinguent selon que ces derniers recherchent avant tout la croissance, de ceux, les plus nombreux, qui désirent d'abord se créer un emploi pérenne qu'ils contrôlent relativement bien, afin de desservir une clientèle proche à satisfaire le mieux possible, en recevant en retour leur achalandage et leurs respects au-delà des simples questions de gains rapides. Comme l'explique Fernand Braudel $\left(1979\right.$; 1985) dans sa grande fresque du capitalisme du Xve au XVIII ${ }^{\mathrm{e}}$ siècle en distinguant ces marchands des commerçants avec leur « amour forcené de l'argent» (Le Goff, 1956, p. 84-85).

La troisième raison provient du fait qu'un des objectifs opérationnels de nos travaux est de conseiller, ou plutôt d'accompagner les entrepreneurs pour aider leurs actions et ainsi leur développement (Schmitt, 2008), soit directement dans nos cours auprès des étudiants dont plusieurs deviendront des entrepreneurs sinon des consultants auprès d'eux, soit indirectement par les résultats des recherches que les firmes-conseils réutiliseront pour appuyer leurs inter-

3 Ou encore Thomas C. Schelling, aussi prix Nobel de 2005.

4 Voir aussi à ce propos la critique récente du groupe de réflexion américain (think tank) appelé Authentic Governance Institute (2014) sur le fait que les grandes écoles de gestion et de droit américaines détruisent la créativité et la capacité de penser de façon holistique. Cet institut explique que l'enseignement y est même devenu « extrêmement formel, bureaucratique et superficiel ». Il est probable que cette critique s'applique aussi à plusieurs de nos facultés de gestion.

5 Ce que notait Adam Smith (1976/1783, p. 142) lui-même en expliquant que « les gens du même métier se rassemblent rarement même pour se divertir et prendre de la dissipation, sans que la conversation aboutisse à une conspiration contre le public, ou à quelque invention pour renchérir leur travail ». 
ventions. Et cette tâche ne peut se faire sans rencontrer des dizaines d'entrepreneurs, discuter avec eux ${ }^{6}$ et visiter leur usine ou leurs bureaux et leur organisation, de façon à mieux les connaître au-delà des théories et des concepts plus ou moins abstraits. Pour cela, il faut souvent recourir à d'autres termes ou formules que ceux utilisés en science si l'on veut se faire comprendre. Comme dans le cas de nos premières enquêtes sur le réseautage, surtout quand nous voulions distinguer chez eux leurs réseaux à signaux forts de ceux à signaux faibles. Ainsi, dans l'étude de 2004 sur l'influence des réseaux sur le dynamisme de leur entreprise, nous avions utilisé les mots " vos contacts au cours des six derniers mois avec des personnes proches ou éloignées de votre entreprise pour discuter des différents aspects de son développement ", en leur demandant de préciser leur origine ou leur emploi et si ces contacts étaient « fréquents » ou «sporadiques" (Julien, Lachance et Morin, 2004) ${ }^{7}$. De même, dans une autre enquête où nous demandions aux propriétaires-dirigeants s'ils partageaient en tout ou en partie leur stratégie avec leurs cadres ou encore si leur organisation était bien impliquée, il était facile de distinguer ceux qui exagéraient ce partage en interrogeant quelques-uns de leurs cadres ou encore cette implication en visitant l'usine avec eux et en observant les comportements des employés (Julien, 2004). Ce qui limite par ailleurs les résultats de trop de travaux basés sur des enquêtes auprès des seuls dirigeants, sauf, probablement, dans le cas des travailleurs autonomes ; même s'il est intéressant de tenir aussi compte de la famille et autres parties prenantes comme la présence d'un mentor officiel ou officieux. Ajoutons que, du point de vue épistémique, ces enquêtes tiennent trop souvent peu compte des biais dus à la présence de l'enquêteur et des orientations données à la recherche ${ }^{8}$.

\section{LES QUATRE FAÇONS DE LIER LA THÉORIE Ȧ LA PRATIQUE}

Il existe au moins quatre façons de faire ce lien entre la pratique et la théorie ou les concepts. La première est l'observation courante. La deuxième porte sur la recherche-action, les études de cas et les enquêtes. La troisième gagne à développer un panel d'entrepreneurs pour discuter avec eux de la valeur de certains concepts. La quatrième demande le développement de liens importants et soutenus avec un groupe d'entrepreneurs.

L'observation régulière rapprochée est facile à faire et devrait constituer l'objet de tous chercheurs en PME et en entrepreneuriat. Elle relève du questionnement discret des comporte-

$6 \quad$ Dans les enquêtes à questions ouvertes auprès des entrepreneurs, il arrive souvent que ces derniers posent eux-mêmes des questions sur certains éléments de leur gestion auxquels ils n'avaient pas réfléchi aussi en détail, en liaison avec certains comportements prônés par la théorie. Cela donne parfois des résultats surprenants issus d'un dialogue qui peut s'installer momentanément et même être renouvelé.

$7 \quad$ Notre pré-test nous avait convaincus qu'en utilisant les mots réseaux et encore plus les différents types de signaux, les entrepreneurs n'auraient pas compris les questions. Il est possible toutefois que, maintenant, ces termes soient plus couramment utilisés chez ces derniers.

8 Ce qui faisait dire à Foerster (2000, p. 66) que «si le principe fondamental du discours scientifique, exige la séparation entre observateur et observé, il est de notre devoir de le transgresser ", même si on se heurte toutefois au problème du solipsisme et des discours déclaratifs. 
ments et décisions entrepreneuriales des petites entreprises auxquelles nous recourons plus ou moins fréquemment dans notre vie quotidienne, comme le garagiste de quartier qui achète un équipement du réglage du train avant pour élargir sa gamme de service ${ }^{9}$, comme le boucher qui fait des paquets plus gros de viande pré-préparée pour répondre à l'arrivée de jeunes familles dans le quartier, comme le poissonnier qui se met à développer des mets préparés pour les clients pressés, comme la coiffeuse qui s'allie à une manucure, comme une ostéopathe qui agrandit son local, ou encore comme le restaurant du voisinage dont nous avons malheureusement prévu qu'il serait en difficulté en constatant qu'il changeait trop souvent son personnel, ce qui est arrivé, etc. Avec de l'empathie et de la finesse langagière, il est facile de discuter avec eux de ces changements, de leur origine et ainsi de leur stratégie, sauf dans le dernier cas. Il est étonnant de voir comment une telle habitude nous permet de saisir toutes sortes de raisons qui vont bien au-delà de la théorie et qui démontrent que les intérêts font intervenir la famille et son cheminement proche ou lointain sinon ses conflits, les problèmes de santé, le développement du quartier, la technologie, la concurrence complexe, etc. ${ }^{10}$ Mon expérience en ce sens a parfois été même suivie d'une discussion ou d'une demande de conseil pour un changement anticipé.

La recherche-action ou les études de cas en profondeur sont des méthodes très intéressantes, mais coûteuses en temps et en ressources pour approfondir et nuancer des concepts. Elles sont toutefois les plus efficaces pour entrer dans le détail de la réalité et en trouver le sens. Elles sont particulièrement propices pour les thèses de doctorat étendues sur quelques années. Elles posent des questions de subjectivité et demandent de prendre diverses précautions bien expliquées dans les livres de méthodologie, même si cette objectivité ne disparaît jamais. Elles ont l'avantage dans les dernières décennies d'être de plus en plus acceptées dans les grandes revues justement parce qu'elles permettent d'analyser en profondeur ces concepts pour les nuancer ou en démontrer la portée limitée. Ajoutons qu'elles peuvent être associées à ce qu'on appelle maintenant le développement du savoir chez les entrepreneurs, dans l'action ou chemin faisant, comme l'expliquent Avenier et Schmitt (2007) ou Schmitt (2008). De leur côté, les enquêtes ont pour objectif de faire des analyses plus généralisables, mais dont la profondeur est limitée par la nécessité de restreindre le temps et donc le nombre et la complexité des questions. Une façon de pallier ça est de multiplier les questions ouvertes, ce qui complexifie toutefois les analyses même si des logiciels d'analyse du discours peuvent aider. Sachant que toute enquête, comme je l'ai dit, a ses biais non seulement du côté du choix et de la forme des questions, mais aussi du moment choisi pour les poser et ainsi des conjonctures de chaque entreprise.

Un meilleur moyen de comprendre le changement dans les entreprises et ainsi la dynamique entrepreneuriale est de suivre un groupe d'entreprises en les associant indirectement à la recherche, notamment en les invitant plus ou moins régulièrement à réagir aux résultats de nouvelles recherches de façon à évaluer la largeur et la profondeur de celles-ci. C'est ce qu'on appelle

9 Cela a entraîné la rupture avec son cofondateur qui ne voyait aucun intérêt et même un risque à élargir la gamme de services, surtout avec un produit demandé peu fréquemment.

10 Un exemple de cette complexité impliquant le long terme est la question du coût élevé du stockage de produits rarement en demande, mais qui étant mal analysé peut favoriser le développement d'un concurrent. Comme ce fut le cas pour une entreprise de pièces usagées d'automobile dans ma ville qui a fermé ses portes onze ans après que je me sois plaint de l'obligation d'aller ailleurs et de la réponse du propriétaire à propos de l'analyse de ses coûts. 
s'associer à un panel d'entreprises ${ }^{11}$. Ce panel peut provenir d'une association d'entrepreneurs souhaitant profiter du savoir des chercheurs pour enrichir leurs propres connaissances. Ce type d'arrangement peut être particulièrement riche s'il est composé d'entrepreneurs aguerris capables de dépasser leurs propres intérêts immédiats pour partager de nouveaux intérêts afin de mieux penser l'avenir. Il suppose cependant des ressources pour communiquer efficacement avec le regroupement, ses membres et les chercheurs, et pour organiser ces rencontres afin que les entrepreneurs y trouvent aussi leur compte.

Finalement, une quatrième façon de faire le lien entre la pratique et la théorie consiste à pousser ces contacts plus loin avec un réseau d'entrepreneurs et de chercheurs travaillant à long terme ensemble pour apprendre réciproquement. C'est l'expérience de la Chaire Bombardier qui a fonctionné de 1994 à 2004 à l'Institut de recherche sur les PME, soutenue par une subvention importante du Conseil national de recherche scientifique, par un apport financier d'un grand donneur d'ordre et par une participation financière des sous-traitants membres, participation qui a augmenté graduellement dans la deuxième phase (Julien, Raymond, Jacob et Abdul-Nour, 2003). Expliquons que cette expérience a démarré dans l'esprit des chercheurs et qu'ils ont fini, après deux ans de discussions difficiles, par la vendre à la direction de la grande entreprise avec l'objectif d'aider à moderniser fortement la production de ses sous-traitants, en particulier du côté de la qualité totale et du recours au système de flux tendus (juste-à-temps). L’expérience était basée sur trois types d'interventions. D'abord, chaque entreprise membre a fait l'objet d'une évaluation détaillée de son organisation et de ses capacités de production, selon une grille d'évaluation sur une échelle ordinale construite à partir des meilleures pratiques connues touchant tous les aspects de fonctionnement d'une très bonne entreprise, en précisant les conditions pour atteindre un niveau supérieur. Ensuite, les chercheurs offraient deux séminaires par année sur les nouvelles pratiques issues des recherches des chercheurs, suivis d'une discussion entre tous les participants ${ }^{12}$. Enfin, certaines interventions complémentaires étaient convenues pour aider les entreprises à s'améliorer là où elles avaient des faiblesses, à moins que le problème soit grave et requière l'intervention d'une firme-conseil.

Les conditions pour faire fonctionner cette chaire étaient d'abord une intervention de la grande entreprise pour inciter leurs meilleurs sous-traitants à travailler avec les chercheurs, ce qui fait qu'une vingtaine d'entre eux, ayant entre 40 et 300 employés, ont graduellement accepté13. Ensuite, le budget permettait la mise sur pied d'un secrétariat pour gérer le tout et l'engagement de plusieurs professionnels de recherche, avec l'appui d'étudiants dans le cadre de leurs travaux

11 Comme l'ont fait nos collègues de l'Université de Nancy, Bayad et Hermann (1991), durant plusieurs années.

12 Les présentations étaient suivies de la réaction d'une entrepreneur-témoin qui avait appliqué dans son entreprise, en tout ou en partie, le ou les concepts discutés dans la présentation; ce qui permettait aux participants de voir et de comprendre plus rapidement les avantages et les limites de la nouvelle pratique et ainsi de générer une riche discussion tant pour les participants que pour les chercheurs.

13 La plus grande difficulté a été de convaincre les cinq premières entreprises poussées par le donneur d'ordre. Ce qui a d'abord exigé deux expériences auprès d'entreprises pour démontrer la capacité des chercheurs à comprendre leurs besoins. Par la suite, ces entreprises ont été les meilleurs « vendeurs » pour attirer les autres firmes. 
appliqués, pour les interventions de plusieurs jours sinon de plusieurs semaines dans les entreprises, sous la supervision des professeurs-chercheurs. De plus, il fallait une équipe multidisciplinaire pour les besoins des entreprises, alors que l'on sait que ces dernières fonctionnent de façon holistique et qu'elles jugent de la qualité des interventions à cette capacité de comprendre, par exemple, qu'un bon problème de production finit irrémédiablement par toucher la formation du personnel, les besoins changeants des clients et ainsi le financement pour la mise à jour des équipements. Enfin, il fallait que les professeurs-chercheurs y trouvent leur propre compte en résultats de recherche, soit pour nuancer les concepts, soit pour en créer de nouveaux, sans oublier la participation aux congrès internationaux pour présenter ces résultats, le tout conditionnant le renouvellement de la subvention du conseil de recherche.

Cela a permis en particulier de répondre aux contraintes majeures restreignant la difficulté de lier la théorie à la pratique à cause des langages, des objectifs et de la gestion du temps trop différents entre les chercheurs et les entrepreneurs. Comme l'a montré entre autres la première recherche sur les PME de l'OCDE en 1989 (Julien, 1993). On sait que les chercheurs recourent à un langage complexe alors que les entrepreneurs utilisent un langage simple. De même, les chercheurs souhaitent obtenir la meilleure réponse, ce qui demande beaucoup de temps en sus des autres tâches, alors que les entrepreneurs veulent une réponse dans quelques mois seulement. Ainsi, si le temps est rarement compté chez les chercheurs, les entrepreneurs savent très bien que s'ils obtiennent la réponse dans huit ou dix mois, les concurrents les auront déjà dépassés. Seule une bonne connaissance des besoins et du temps requis permet d'ajuster les possibilités de recherche et de construire une relation de confiance et de connaissance réciproques pour une collaboration efficace pour les deux parties.

Tout ça, c'est finalement faire de l'abduction comme l'expliquait Peirce, mais aussi Platon, ce que les chercheurs états-uniens ont redécouvert avec la théorie ancrée pourtant vieille comme le monde. Comme trop de concepts anciens qu'ils réinventent avec des mots nouveaux, comme l'innovation ouverte, l'effectuation (concept utilisé par Deleuze) comme le bricolage qui a presque toujours existé chez les petits créateurs d'entreprises achetant du matériel usagé et aux ressources très limitées, et combien d'autres!

Malheureusement, ils oublient ainsi Tocqueville qui pourtant avait vanté dans son ouvrage les avantages de la jeunesse des États-Unis et de leur organisation politique décentralisée et participante. Lui qui a passé près d'une année à visiter la plupart des grandes villes états-uniennes de l'époque pour mieux en comprendre la philosophie et les pratiques de cette population ; population qui avait fui et ensuite combattu le régime royal anglais pour finalement mettre en pratique les principes de la Révolution française. Gilles Deleuze (1991, p. 106), délaissant les concepts abstraits pour mieux interroger la vraie vie, n'explique-t-il pas aussi la même chose : "Penser, c'est expérimenter. Mais l'expérimentation, c'est toujours ce qui est en train de se fairele nouveau, le remarquable, l'intéressant»? 


\section{RÉFÉRENCES}

Authentic Governance Institute (2014). Higher education system sucks. Récupéré le 20 septembre 2015 du site : http://goo.gl/2SBYjZ.

Avenier, M.-J. et Schmitt, C. (2007). La construction de savoirs pour l'action. Paris, L'Harmattan.

Bayad, M. et Hermann, J.-L. (1991). Panel PMI lorraines. Rapport technique de la seconde vague. Nancy, Centre régional de documentation en gestion.

Bergson, H. (1907/1969). L'évolution créatrice. Paris, Presses universitaires de France.

Braudel, F. (1979). Civilisation matérielle, économie et capitalisme, XV $V^{e} X V I I I^{e}$ siècle. Paris, Armand Colin.

Braudel, F. (1985). La dynamique du capitalisme. Paris, Arthaud.

Deleuze, G. (1991). Qu'est-ce que la philosophie ? Paris, Éditions de Minuit.

Foerster, H. (2000). Éthique et cybernétique de second ordre. Dans P. Watzlawick et G. Nardone (dir.), Stratégie de la thérapie brève (p. 60-76). Paris, Seuil.

Julien, P.-A. (1993). Petites et moyennes entreprises. Compétitivité et technologies. Paris, OCDE.

Julien, P.-A. (2004). Actionnabilité de la recherche universitaire : du temps long au temps allongé. Le cas de la Chaire Bombardier. Revue internationale PME, 17(3-4), 69-94.

Julien, P.-A., LACHAnCE, R. et Morin, M. (2004). Signaux forts et signaux faibles : une enquête sur les liens réticulaires dans les PME dynamiques. Géographie, économie et société, 6(2), 179-202.

Julien, P.-A., Raymond, L., Jacob, R. et Abdul-Nour, G. (2003). L'entreprise-réseau. Dix ans d'expérience de la Chaire Bombardier. Québec, Presses de l'Université du Québec.

Le Goff, J. (1956). Marchands et banquiers du Moyen Âge (collection «Que sais-je?»). Paris, Presses universitaires de France.

Mintzberg, H. (1994). The rise and fall of strategic planning. Londres, Prentice Hall.

Peirce, C.S. (1896/1994). Le raisonnement et la pratique des choses. Paris, Cerf.

Popper, K. (1965). Conjectures and refutations (2 édition). New York, Basic Books Publishers.

Smith, A. (1776/1783). Recherches sur la nature et les causes de la richesse des nations. Londres, Pierre J. Duplain.

Schмiтt, C. (2008). Université et entrepreneuriat. Nancy, Presses de l’Université de Nancy.

Schmitт, C. (2015). Réconcilier action et connaissance : l'agir entrepreneurial. Québec, Presses de l'Université du Québec (à paraître). 